\title{
RECENSIÓN: TERAPIA OCUPACIONAL Y EXCLUSIÓN SOCIAL
}

\author{
Palabras Clave: \\ Terapia ocupacional, exclusión social, inclusión social, derechos humanos, globalización, intervención social, intervención comunitaria, \\ paradigma social de la ocupación.
}

\section{Rodolfo Morrison J ${ }^{1}$.}

Frank Kronenberg propone en el prólogo del texto Terapia Ocupacional y Exclusión Social de Navarrete, Cantero, Guajardo, Sepúlveda y Moruno (2015) diferentes posibilidades y cuestionamientos respecto a la terapia ocupacional (en adelante TO). Señala que la disciplina se encuentra en un nuevo paradigma donde se hace necesario revisar sus bases y estándares sobre la formación profesional. Se nos plantea en este prólogo que los derecho humanos (en adelante $\mathrm{DDHH}$ ) son una parte crucial de la TO y que las "epistemologías del sur", citando a Sousa Santos, han permitido fundamentar: "prácticas y racionalidades emergentes" (p. 10) en la disciplina. En este contexto, este libro resulta fundamental para propiciar las discusiones dentro de la $\mathrm{TO}$ y la Ciencia de la Ocupación (en adelante CO).

Rápidamente, en la introducción, la autora y los autores realizan una propuesta respecto a la comprensión de las diferentes problemáticas que experimentan usuarias y usuarios de TO, señalan que: "las dificultades con las que tropiezan millones de personas en todo el mundo para desarrollar sus ocupaciones, encuentran su explicación en las condiciones históricas, estructurales en las que ellas se producen y manifiestan" (p. 16). Así, proponen que: "la terapia ocupacional, desde sus

1 Doctor y Máster en Lógica y Filosofía de la Ciencia (Univ. de Salamanca), Licenciado en Ciencias de la Ocupación, Terapeuta Ocupacional (Univ. Austral de Chile). Académico y coordinador del área Investigación del Departamento de Terapia Ocupacional y Ciencia de la Ocupación, Facultad de Medicina, Universidad de Chile. Integrante de la Sociedad Chilena de Ciencia de la Ocupación (SoChCO) y del Comité de Estudios en Ciencia de la Ocupación (COECO) de la Universidad de Chile, rodolfomorrison@med.uchile.cl campos más tradicionales (...) y por razones histórico sociales (...) ya estaba siendo convocada a resituar lo ocupacional y sus métodos de trabajo hacia el terreno de lo social y lo colectivo" (p. 18-19). Esto justifica, de alguna manera, que la TO se involucre de lleno en campos vinculados a "lo social", aún cuando, podríamos considerar que "lo social" siempre ha sido inherente a la profesión, principalmente desde sus inicios, los que sin embargo han sido olvidados (Morrison, 2013), pero recuperados por aportes como el de Navarrete, Cantero, Guajardo, Sepúlveda y Moruno.

De esta manera, hacen hincapié en: "[n]uestra profesión debe ser capaz de poner en el centro de su quehacer los $\mathrm{DDHH}$, desde una perspectiva colectiva y comunitaria y que se oriente hacia la justicia y la inclusión social." Y continúan: "No sólo eso, debe ser capaz de comprometerse críticamente a través de la educación, la investigación y la práctica para tratar de eliminar las desigualdades" (Navarrete et al., 2015, p. 20). Este párrafo nos deja entrever sobre qué temas tratará el libro, sin embargo, no es hasta el final de la introducción cuando hacen explícitos sus objetivos. Primero, reflexionar sobre los enfoques teóricos que dan soporte a la profesión en contextos de exclusión social y $\mathrm{DDHH}$, y segundo, proponer orientaciones técnicas, por medio de la sistematización de intervenciones exitosas, para la práctica disciplinar. ¿Logran Navarrete y los demás autores tal meta? Veamos.

En el desarrollo del capítulo I aparecen diferentes temas, uno de ellos vinculado a la globalización y sus consecuencias desde una mirada neoliberal, en la que el capitalismo se desarrolla generando nuevas formas de producción, cambios en el mundo del trabajo, el medio ambiente y el rol del Estado, además de la generación y reproducción de la vulnerabilidad social, cuyos productos son "la mercantilización de la educa- 
ción, la salud y la seguridad social" (p. 25). En este contexto, se nos propone que la TO, más que enfocarse en la igualdad, debe centrarse en el derecho a la diferencia en colaboración con actores y movimientos sociales.

Alejarse del individualismo, como lo han propuesto recientes movimientos de la $\mathrm{CO}$ (Whiteford \& Hocking, 2012), y considerar a las personas como: "sujetos colectivos que en sus prácticas ocupacionales se constituyen en personas de derecho" (Navarrete et al., 2015, p. 26), rescatando lo diverso, lo diferente y lo participativo. "En el fondo, se propone la TO como una práctica política social transformadora y no reproductora de sistemas sociales alienantes y opresores" (p. 26).

En el capítulo II, otro de los aspectos resaltados es lo social y lo comunitario en la TO. Aquí se revisa, a modo de marco teórico, diferentes elementos fundamentales para comprender el rol de la TO en este nuevo paradigma, que podríamos suponer como el Paradigma Social de la Ocupación (Morrison, Olivares y Vidal, 2011). Así, se refuerza la idea de la exclusión social y la justicia social como elementos centrales de la disciplina y se cita a Manfred Max Neef para señalar cómo la existencia de pobrezas, y no de una pobreza, como pobreza de subsistencia, de protección, de afecto, de entendimiento, de participación, de identidad, entre otras, son elementos teóricos que la TO debe incorporar como antecedentes para su ejercicio profesional. Así, la autora y los autores proponen a las justicias, como planteamientos teóricos, pero vinculados inherentemente a los DDHH.

De este modo, distinguen tres generaciones de $\mathrm{DDHH}$. Los primeros fundados en el valor de la libertad, como el derecho a la vida, la seguridad, la igualdad ante la ley, la prohibición de la tortura y los tratos crueles, a la libre circulación, ente otros; los segundos vinculados con el derecho al trabajo, la remuneración equitativa, el descanso, la asistencia médica, el derecho al tiempo libre, etc.; y los terceros relacionados con el medio ambiente, la paz, el patrimonio común, entre otros. Estos últimos implican concebir a las personas, no como individuos, si no como sociedad civil: "pueblos como poseedores de derecho y con ello las personas que ahí habitan" (Navarrete et al., 2015, p. 36). Así, los DDHH-que son objetivos al ser configurados como un producto de las relaciones humanas concretas e históricas - operan en: "un campo de relaciones históricamente dadas en las cuales se produce la vida concreta de los sujetos, vida primariamente cultural, simbólica, política, y ética" (p. 38).
Por otro lado, otro elemento relevante en el texto es el de ciudadanía, comprendida como justicia, reconocimiento, autodeterminación y solidaridad, y como elemento primario de las personas -no como un logro a alcanzar, si no a desempeñar, ya que se es ciudadano per se-, es amenazada por el concepto de ciudadanía liberal, que se centra en el "atomismo individual" y no en la mirada social. Entonces, se nos invita a reconocer a lo social como un principio ontológico, a lo social como: "un campo relacional históricamente constituido, situado, concreto, con relaciones económicas sociales específicas, con una cultura particular" (p. 40). De esta manera, la ciudadanía es inherente a las relaciones sociales, ciudadanía son los sujetos.

Otros elementos teóricos del texto, como la educación popular, el concepto de empoderamiento, fortalecimiento y la rehabilitación de base comunitaria son abordados de manera general estableciendo algunas relaciones con la disciplina. Específicamente, vinculan el trabajo comunitario de la TO con elementos como la participación, el autocontrol y la autogestión que deben estar presentes en toda intervención social.

Después de esta interesante propuesta teórica general, se realiza un abordaje desde la propia disciplina. Es así como, en el capítulo III, aparecen conceptos como la justicia ocupacional (en adelante JO), quien toma el protagonismo, apoyada por referencias a autores como Wilcock, Kronenberg, Pollar y Simó. Al vincularse con el área de la salud, promover la JO es una tarea de la disciplina, pero también prevenir la marginación ocupacional y otros factores de riesgo. De esta manera, el rol político de la TO toma fuerza, en especial al considerar las actividades políticas de la vida diaria. Así, las y los terapeutas ocupacionales somos parte del problema de la exclusión social y por ende, podríamos ser parte de la solución.

Al finalizar este capítulo nos puede quedar la sensación de que los elementos disciplinares no tienen el mismo peso teórico que los otros señalados anteriormente, lo que podríamos interpretar como una invitación y provocación a continuar con su desarrollo. De esta manera, la autora y los autores proponen: "[u]na práctica de TO basada en los DDHH y centrada en la inclusión social de personas vulneradas con estrategias socio-comunitarias, implica la construcción de un nuevo orden de fundamento disciplinar y profesional" y continúan: "[c]onlleva a reflexiones de diferente orden (epistemológicas, metodológicas, éticas y políticas) que nos ponen en un plano de ruptura con las miradas tradicionales y dominantes en la disciplina (...)" (p. 57). 
Es así como, en la segunda sección del libro, se nos proponen diferentes experiencias en Chile, Burkina Faso y España. Primero, Cifuentes, Espejo y Jacard relatan su experiencia en un programa de rehabilitación psicosocial y Pérez sistematiza la intervención de TO en personas en situación de calle. En estas experiencias, aunque se señalan elementos teóricos interesantes, en especial en el segundo relato, donde el sentido de los DDHH retoma su protagonismo al buscar "condiciones mínimas de calidad de vida" (p. 88) y combatir la exclusión, vemos algo de distancia entre los elementos teóricos señalados en la primer sección del libro y las reflexiones de estos autores. Ello nos podría hacer pensar sí la disciplina está preparada para asumir este nuevo cambio de paradigma, sin embargo, poco a poco podemos ver un tránsito interesante, tanto en el uso del lenguaje, como en las reflexiones teóricas de estos autores. Aspectos que se resaltan en estos trabajos son el trabajo en red, comprender al sujeto colectivo como conceptualización esencial para el logro de objetivos en este contexto, además de elementos de inclusión social y los DDHH como hemos señalado.

La tercera experiencia chilena, a cargo de Schliebener trata sobre el tiempo libre en personas que se encuentran en tratamiento por consumo problemático de alcohol y drogas. Desde aquí en adelante, emerge un vinculo más estrecho entre la primera y segunda sección del texto. As así como, la autora propone que las reflexiones en la practica profesional se orienten a que: "los usuarios de esta terapia grupal, conozcan, discutan y comprendan su posición como sujetos sociales dentro de una sociedad donde (...) [el] uso del tiempo libre (...) [se orienta] hacia la satisfacción rápida de placeres (...) [como] el consumo de sustancias y (...) productos en el mercado" y continúa señalando que este hecho: "permite alejarse de las vivencias del tiempo de trabajo que está presente en la mayor parte de la rutina de las personas" (p. 107). De esta manera, el objetivo es claro: "Es así como no sólo se facilita un espacio de cambios de uso del tiempo libre de forma mecánica (...), sino que se abren canales de conciencia, donde los usuarios se autoidentifiquen (...) como agentes de cambio social (...) (p. 108).

Los principios sobre DDHH, ciudadanía y JO son coherentes con el enfoque propuesto. Experiencias concretas como ir al cine o al teatro se problematizan desde una mirada política o vinculada al rol del Estado en la exclusión social y reproducción del consumo problemático. La autora recoge los relatos de sus usuarios, señalando su preocupación por verse obligados a "encerrarse en sus hogares", ya que, desde su percepción, las opciones de actividades de tiempo libre se vinculan al uso de dinero o asociadas al consumo de sustancias, lo que pone en riesgo su tratamiento. Paulatinamente, comienzan a surgir alternativas, luego de la problematización, como sujetos colectivos. Una de las manifestaciones más claras es la creación de un boletín informativo, donde los usuarios señalan: "[e]n esta oportunidad queremos difundir la gran problemática que es para nosotros el uso del tiempo libre. Porque no tenemos acceso a espacios (...) protegidos del consumo de alcohol y drogas". Claramente intentan hacer partícipe a la comunidad como parte del problema y la solución, pero también al Estado y otras instituciones.

La cuarta experiencia, relatada por Zango, presenta la sistematización del primer centro de TO en Burkina Faso (África). Aquí se continúa en la profundización de elementos teóricos disciplinares y se destaca el valor de la cooperación internacional para combatir la exclusión social, además del rol de la comunidad como co-responsables de la salud mental de sus integrantes. Zango nos muestra lo complejo de muchas políticas sociales, por ejemplo, en Burkina Faso aunque no son prioritarias las temáticas de salud mental (realizándose énfasis en temáticas como el VIH), se ha demostrado que: "las personas afectadas por la enfermedad mental son más vulnerables a comportamientos de riesgo para su salud y tienen más dificultades de seguir un tratamiento médico en caso de padecer una enfermedad" (p. 137). Esto nos da cuenta de que la inclusión social debe ser apoyada desde macrosistemas que repercuten a nivel local, o bien, desde sistemas mediatos, expresados en políticas públicas que tengan expresiones en los cotidiano (Kronenberg, Simó \& Pollard, 2006).

Burkina Faso es uno de los países más pobres del mundo, por desconocimiento algunas personas con enfermedad mental son expulsadas de sus familias y hasta envenenadas por riesgo a reiterar episodios agudos. Por ende, un centro de TO debe tener relevancia cultural y estar en sintonía con las necesidades e intereses de la población. Además, Zango propone que: "[d]esde el punto de vista teórico esta experiencia es un reto para indagar sobre la conceptualización de la ocupación (...) que como terapeutas ocupacionales con una formación occidental estamos acostumbrados a utilizar" (Navarrete et al., 2015, p. 167).

Finalmente, la quinta sistematización es española y corresponde a los aportes de la TO en la población gitana en riesgo de exclusión social. Su autor es Emeric, quien comienza proponiendo la relevancia que tiene el 
contacto directo con las poblaciones vulnerables por medio de un diagnóstico situacional que permita evidenciar las problemáticas reales de las personas y alejadas de prejuicios sociales. De esta manera, se vislumbran elementos como el estigma social, que limita los procesos de inclusión, así: "[e]xiste (...) una mayoría gitana incorporada efectivamente a una vida cotidiana en sociedad, invisibilizada por las problemáticas mediatizadas que afectan a una minoría más vulnerable, produciéndose un doble (...) proceso de exclusión" (p. 183). Emeric se refiere a las personas que pasan desapercibidas por un sistema que niega o restringe oportunidades y de aquellas personas que poseen carencias en redes de apoyo al generarse categorías de ciudadanos. Por ende, las acciones a desarrollar se vinculan con procesos de intervención situados que recojan las experiencias de las personas.

Emeric señala que en este contexto: "debe promoverse la investigación y el desarrollo de modelos y herramientas propias de TO con mayor capacidad adaptativa para el trabajo en y con la comunidad" (p. 206). Así, este último capítulo, de cierta forma, sintetiza las principales preocupaciones del texto, pero abre una pregunta para quienes observamos estas prácticas sociales de la terapia ocupacional.

¿Cómo vincular una mirada social crítica dentro de problemáticas concretas? ¿Es posible hablar de "intervención social", o de generación de cambios sociales, en un contexto capitalista que promueve la segregación social y la exclusión? Y finalmente, ¿qué obligación tiene la TO en este contexto? El texto de Navarrete, Cantero, Guajardo, Sepúlveda y Moruno nos entrega luces para seguir un camino que posiblemente nos permita desarrollar algunas de estas respuestas.

Es así como la meta de la autora y los autores es alcanzada. Y aunque podamos pensar en que la TO social representa un campo nuevo de acción, es importante recordar que ha sido (y probablemente será) una parte inherente de la disciplina y de nuestro rol profesional, el que quizás, podríamos sintetizar así: colaborar en que este mundo sea un lugar mejor para todos y todas.

\section{BibliografíA}

Kronenberg, F., Simó, S. \& Pollard, N. (2006). Terapia Ocupacional sin Fronteras: aprendiendo del espíritu de los supervivientes. Buenos Aires: Ed. Médica Panamericana.

Morrison, R. (2013). ¿Por qué necesitamos mirar hacia atrás? Volviendo a lo esencial: un enfoque epistemológico al "árbol de la terapia ocupacional". TOG (a Coruña). Disponible en: http://www.revistatog. com/num18/pdfs/original4.pdf [Consultada el 20 de abril de 2015]

Morrison, R., Olivares, D., \& Vidal, D. (2011). La filosofía de la Ocupación Humana y el paradigma social de la Ocupación. Algunas reflexiones y propuestas sobre epistemologías actuales en Terapia Ocupacional y Ciencias de la Ocupación. Revista Chilena de Terapia Ocupacional, 11(2), 102-119. doi:10.5354/0717-5346.2011.17785

Navarrete, E., Cantero, P., Guajardo, A., Sepúlveda, R. \& Moruno, P. (2015). Terapia Ocupacional y Exclusión Social: Hacia una praxis basada en los derechos humanos. Charleston, SC: Ed. Segismundo.

Whiteford, G. \& Hocking, C. (2012). Occupational Science: Society, Inclusion and Participation. Australia: John Wiley \& Sons. 